\title{
PReS-FINAL-2265: Tuberculosis in pediatric patients who are receiving anti-TNF agents
}

\author{
J Calzada-Hernández ${ }^{*}$, A Noguera Julian², S Ricart Campos , R Bou Torrent ${ }^{1}$, E Iglesias Jiménez¹, \\ MI González Fernández ${ }^{1}$, J Sánchez Manubens ${ }^{1}$, V Torrente Segarra ${ }^{1}$, L Rozas Quesada², FJ Martín Carpi ${ }^{3}$, \\ J Antón López ${ }^{1}$
}

From 20th Pediatric Rheumatology European Society (PReS) Congress

Ljubljana, Slovenia. 25-29 September 2013

\section{Introduction}

Adult patients receiving anti-TNF $\alpha$ treatment are at increased risk for developing tuberculosis (TB). Few data have been published in the pediatric population.

\section{Objectives}

We describe the occurrence of latent tuberculosis infection (LTI) and TB in children and adolescents treated with anti-TNF $\alpha$ agents.

\section{Methods}

Cohort observational study including pediatric patients receiving anti-TNF $\alpha$ agents in a tertiary-care pediatric hospital. LTI is ruled out by the implementation of antiTNF $\alpha$ drugs by tuberculin skin test (TST) and, from March 2012, QuantiFERON Gold-In Tube ${ }^{\circledR}$ test (QTF). Along treatment, patients are evaluated periodically for TB using history and physical examination, but TST/ QTF are not systematically repeated.

\section{Results}

The final cohort consisted of 261 anti-TNF $\alpha$ treatments in 221 patients (56.1\% female), of whom $51.7 \% / 31$. $\% / 17.2 \%$ treated with etanercept/adalimumab/infliximab, respectively, for a variety of rheumatic diseases (75.6\%), inflammatory bowel disease $(20.8 \%)$ and inflammatory eye diseases $(3.6 \%)$. The mean(SD) age at diagnosis of the primary condition was 7.2(4.6) years and the duration of the disease before implementing the anti-TNF $\alpha$ agent was 3.0(3.3) years. The total follow-up time under anti-TNF $\alpha$ treatment was 614 patients-year; mean(SD) time per patient: $2.8(2.2)$ years.

Pediatric Rheumatology Unit, Pediatrics Department, Hospital Sant Joan de Déu, Esplugues de Llobregat (Barcelona), Spain

Full list of author information is available at the end of the article
LTI was diagnosed in 3 adolescent girls (prevalence rate: $1.4 \%$; $95 \%$ CI: $0-2.9$ ) affected with juvenile idiopathic arthritis, who received isoniazid chemoprophylaxis and were later treated with anti-TNF $\alpha$, without incidences. QTF tested positive in all three patients, while TST was positive in only one of them. No incident cases of TB were observed.

\section{Conclusion}

In our study, the prevalence of LTI (1.4\%) was similar to that reported in population screening studies in Spain and no incident cases of TB were observed.

\section{Disclosure of interest}

None declared.

\section{Authors' details}

'Pediatric Rheumatology Unit, Pediatrics Department, Hospital Sant Joan de Déu, Esplugues de Llobregat (Barcelona), Spain. ${ }^{2}$ Pediatric Infectious Diseases Unit, Pediatrics Department, Hospital Sant Joan de Déu, Esplugues de Llobregat (Barcelona), Spain. ${ }^{3}$ Pediatric Gastroenterology Department, Hospital Sant Joan de Déu, Esplugues de Llobregat (Barcelona), Spain.

Published: 5 December 2013

doi:10.1186/1546-0096-11-S2-P255

Cite this article as: Calzada-Hernández et al:: PReS-FINAL-2265:

Tuberculosis in pediatric patients who are receiving anti-TNF agents. Pediatric Rheumatology 2013 11(Suppl 2):P255. 